\title{
LETTERS
}

\section{Joint study led by International Food Policy Research Institute (IFPRI) puts meat-eating women's lives at risk}

Published online first on August 8, 2020 DOI: 10.20529/ IJME. 2020.084

Keywords: Nutrient adequacy, diet during pregnancy, vegetarian diet, food diversity

The findings of a collaborative study in Uttar Pradesh, India, by the International Food Policy Research Institute (IFPRI), Family Health International (FHI) and Johns Hopkins (JH), and funded by the Bill and Melinda Gates Foundation (BMGF), have been tweeted out from the IFPRI Twitter handle (See: https://twitter. com/POSHANsm/status/1268074050468712449) on June 4, 2020, along with a graph, stating;

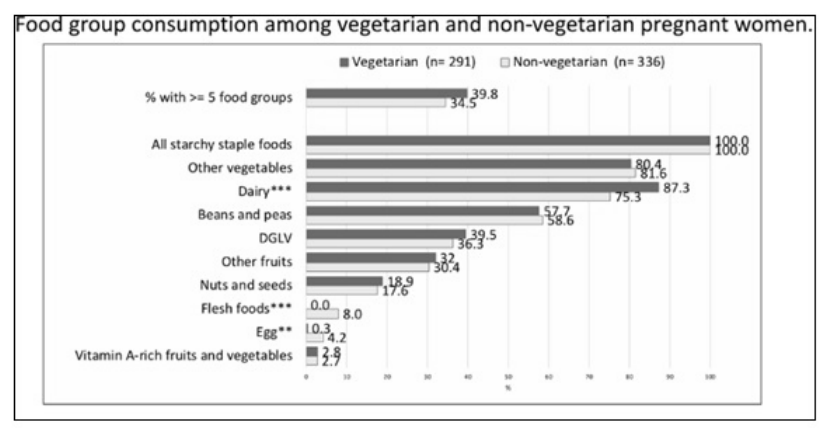

Source: IFPRI twitter handle

'Vegetarian women more likely to have probability of nutrient adequacy and diet diversity during pregnancy than nonvegetarian women-

The next tweet claims that this study 'is a finalist for the Emerging Leaders in Nutrition Science Award-

On enquiry, another tweet was put out 24 hours later, with the addition of the text in bold.

'Vegetarian women were more likely to have probability of nutrient adequacy and diet diversity during pregnancy than nonvegetarian women, but these differences are likely confounded by socio-economic and caste status".

We are shocked by this study as well as the way it has been projected in the public domain, for the reasons stated below:

"Vegetarian" and "non-vegetarian" are not scientific categories. In the current politically and ideologically charged environment of India, specifically in the state of Uttar Pradesh, even somewhat sensitive researchers would have been conscious of the ethical pitfalls and dangers of this kind of a nutritionally irrelevant discourse. Predictably, the projected "results" show up the confusions and contradictions.

This study shows that only $8 \%$ of self-reported non-vegetarian women consumed flesh foods and only $4.2 \%$ consumed eggs. Although, this is not representative of meat eating statistics of the rural population in India, the authors make no attempt to explain this glaring inconsistency. The finding that "nonvegetarians are not eating enough animal foods"should disturb anyone concerned about nutrition. Instead of being concerned about this important finding which highlights that only $8 \%$ of non-vegetarians actually get to eat some meat, the authors turn the findings on their head and try to appear on the right side of the political dispensation by projecting that vegetarians have more food diversity

Animal proteins are important for iron absorption and animal foods are a good source of Vitamin B12, Vitamin A and Riboflavin. In addition, fish is a rich source of N3 fatty acids. These nutrients are especially vital during pregnancy because almost $50 \%$ of pregnant women are known to be anaemic with multiple nutrient deficiencies.

As Natarajan and Jacob (1) point out, "self-identification" into the categories of vegetarian/non-vegetarian is not reliable because even meat-eating people may self-identify as "vegetarian" out of social pressure. For instance, if children in government schools where Akshaya Patra provides food were asked about what they eat at home, they are likely to say that they eat 'sattvik food without onion and garlic" 1. This could be attributed entirely to the current social and political pressures on eating choices, irrespective of what children eat or enjoy eating in reality.

As any well trained nutritionist would know, dietary diversity is one aspect of nutrition, but it cannot be taken in isolation while ignoring portion size, adequacy and nutrient density of food, especially in pregnancy. Several studies use a cut-off of $15 \mathrm{~g}$ for a food to be considered for dietary analysis. No effort has been made in the tweets put out by IFPRI to indicate what the cut-off quantity is and whether this led to exclusion of certain foods in the analysis. To give an example of nutrient density, $15 \mathrm{gm}$ of meat or egg is not equivalent to $15 \mathrm{gm}$ of cereal, so not representing these differences shows poor methodology and even poorer understanding of nutrition. 
Since this is a study about pregnancy, it should also be known that pregnant women in India have dismally low pre-pregnancy weights and heights, poor weight gains and anaemia during pregnancy, resulting in low birth weights and post-partum complications, all closely related to consumption of a monotonous cereal pulse diet bereft of good quality protein, iron, and calcium. To repeat, focusing only on diversity while ignoring adequacy and nutrient density defeats the purpose of studying nutrition intakes in pregnancy.

Women from better socio-economic background, are likely to have more food diversity with more access to animal foods like dairy. Women from dominant castes are also likely to be from a better socio-economic background, which in turn can contribute to better pregnancy outcomes. Martin-Prével et al (2), observed that women of reproductive age who consume five or more food items are also highly likely to consume at least one animal-source food. Having being co-authors with Martin-Prével on other studies, it is inexplicable how the researchers from IFPRI have overlooked this point and reach the conclusion that vegetarians have more diversity.

The dietary data for this study comes from pregnant women enrolled at baseline from an Alive and Thrive maternal nutrition programme in Uttar Pradesh, India - a project funded by the Bill and Melinda Gates Foundation. Incidentally, BMGF has also funded this study. Considering the results of the study, it is surprising that these women have not benefitted from the BMGF project. Is it possible that the project itself has become a hostage to promoting vegetarianism in UP? In addition, being a population under a specific project, this group of women cannot be taken to represent the general population and especially not for the purpose of making sweeping conclusions about "vegetarian and non-vegetarian" women.

The researchers claim that median intake of micronutrients was below the estimated average requirement (EAR) for 9 out of 11 micronutrients, with only zinc and thiamine having a median intake slightly above EAR. Not surprisingly the results show that intake is low in both categories. This is a classic example of the pitfalls of relying on oral questionnaires for nutrition data collection in an atmosphere of fear and marginalisation of meat eaters. Even a rigorously conducted 24 -hour oral recall method can show variations of up to $25 \%$. Biochemical estimations may have given us some insights into the actual intakes in the two groups

We are shocked by the fact that results of studies with potential large impact on women, especially pregnant women, are put out on Twitter like slogans or sensational headlines. Sharing teasers on social media while withholding the full study wouldn't fall into the category of serious or ethical research.

This valorising of vegetarianism and dismissal of meat eating is not isolated, but rides on the back of several years of false assertions that India is "vegetarian". There has been criminalisation of meat (particularly beef) eating communities, vehement denial of eggs to children (who are often some of the poorest, most vulnerable and malnourished) in spite of the Right to Food being a legal mandate.

Blatant promotion of religious, sattvik, casteist, vegetarian institutions like Akshaya Patra irrespective of serious objections by public health experts, food rights activists, doctors, researchers, are symptoms of the imposition of a majoritarian view in India. This is made worse by the growing global push towards predominantly plant based diets through multilateral agencies like the Eat Lancet Commission and promoted by Indian counterparts, in spite of global criticism. This "study" is therefore not unbiased.

It is alarming that IFPRI, Johns Hopkins, FHI and BMGF are coming together to put out the message on social media that vegetarian food is superior - a pre-existing casteist and anti-minority myth in India. That this study is a finalist for the Emerging Leaders in Nutrition Science award, is probably the icing on this vegetarian cake.

We do hope that the authors will rethink about publishing this study in the present form.

${ }^{1}$ Note: A sattvik diet is a brahmanical vegetarian diet

Sylvia Karpagam (sakie339@gmail.com), Public health doctor and Researcher, Bengaluru Karnataka, 560 047, INDIA; Veena Shatrugna (veenashatrugna@yahoo.com), Retired Deputy Director, National Institute of Nutrition, Hyderabad, Telangana, INDIA.

Editorial Conflict of Interest: The editor did not participate in the processing and decision making on this manuscript as he is one of the signatories to the letter.

\section{References}

1. Natarajan B, Jacob S. Putting Indian food habits in their place. 'Provincialising' vegetarianism. Econ Pol Wkly. 2018 March 3; 53(9). Available from: https://www.epw.in/journal/2018/9/special-articles/ provincialising-vegetar ianism.html\#: :tex $\mathrm{t}=$ Large $\% 2$ Dsc ale\%20 sur v e y\%20da ta\%20ar e,b y\%20c ommon\%20claims\%20and\%20 stereotypes.

2. Martin-Prével $Y$, Allemand $P$, Wiesmann $D$, Arimond $M$, Ballard $T$, Deitchler M, Dop MC, Kennedy G, Lee WT, Mousi M. Moving forward on choosing a standard operational indicator of women's dietary diversity.Washington DC: IFPRI; 2015 [cited 2020 Jul 11]. Available from: https://www.ifpri. org/publication/moving-for ward-choosingstandard-operational- indicator-womens-dietary-diversity

[This letter was endorsed by 148 activists, researchers, public health professionals, doctors and concerned citizens and first uploaded at: https://aharanammahakku.home. blo g/2020/06/16/sta t emen t-in t er na tional-f o o d-p olic yresearch-institute-ifpri-family-health-international-fhi-andjohn-hopkins-put-meat-eating-womens-lives-at-risk/ It was sent to the authors of the study on June 18,2020.] 